\title{
Neuroimagen y neurotoxicidad inducida por MDMA (éxtasis)
}

\author{
Una D. McCann*; George A. Ricaurte** \\ *Departamentos de Psiquiatría **Departamento de Neurología: Johns Hopkins School of Medicine
}

Enviar correspondencia: Una D. Mc Cann. The Johns Hopkins School of Medicine. Baltimore, MD 21224. E-mail: umccann@helix.nih.gov

\begin{abstract}
Resumen
El incremento del consumo de éxtasis (MDMA) por parte de los jóvenes ha obligado al estudio de efectos a corto y medio plazo sobre el SNC. En la última década, se demostró la existencia de neurotoxicidad serotoninérgica en el SNC. Recientemente aparecieron estudios tendentes a demostrar también daño dopaminérgico que se ha evidenciado en experimentación animal. Las diferentes técnicas de neuroimagen han contribuido a estos hallazgos, a través del estudio de flujo como del nivel de captaciones en los distintos sistemas receptoriales implicados, tanto presinápticos como postsinápticos. Los resultados que se compilan proceden de los trabajos de neurotoxicidad más relevantes realizados a través de las diferentes técnicas de neuroimagen (SPECT, PET, MRS, MRI de difusión y perfusión). Se concluye que existe suficiente demostración, en diferentes especies animales, incluidos primates, de que la MDMA produce neurotoxicidad serotoninérgica y dopaminérgica y que existen resultados clínicos que confirman estos hallazgos en humanos.
\end{abstract}

Palabras claves: éxtasis, MDMA, neurotoxicidad, neuroimagen.

\section{Summary}

The increase in the use of ecstasy (MDMA) by young people has let to the study of short and long term effects on the CNS. The existence of serotonergic neurotoxicity has been demonstrated in the last decade. Recent animal studies, including primates, demonstrate and clinical findings suggest dopaminergic damage. Different neuroimaging techniques have contributed to these findings through the study in the different receptor systems involved both presynaptic and postsynaptic levels. The results are obtained from the most relevant studies of neurotoxicity by means of the different neuroimaging techniques (SPECT, PET, MRS, diffusion and perfusion MRI). Considerable evidence from different animal species, including primates, exists that MDMA produces both serotonergic and dopaminergic neurotoxicity. Recent evidence has been published which confirm this findings in humans.

Key Words: ecstasy, MDMA, neurotoxicity, neuroimaging.

\section{INTRODUCCIÓN}

$\mathbf{E}$ I consumo de la droga psicoactiva, $( \pm)$ 3,4-Metilenodioximetanfetamina (MDMA, "Extasis") se ha incrementado de una manera muy significativa durante la última década (Johnson et al., 2001a; 2001b; Christophersen, 2000). Resulta extra- ordinario que el elevado incremento del consumo de MDMA se haya producido a pesar de que ya durante las dos últimas décadas se conociera que la MDMA es una potente neurotoxina serotoninérgica para los animales. De hecho, se ha demostrado, en animales tratados con MDMA, una reducción persistente de serotonina (5-HT) en axones de neu- 
ronas serotoninérgicas, que se deben, según estudios anatómicos, a la axotomía distal de los mismos (para información, ver Ricaurte et al., 2000).

Hasta hace poco, la opinión más aceptada era que la MDMA era una neurotoxina altamente selectiva, produciendo daños en las neuronas cerebrales 5-HT, pero sin afectar a otros tipos neuronales. Esta opinión se basaba en una serie de estudios que indicaban que la dopamina cerebral (DA) y las neuronas noradrenérgicas no se veían afectadas por las dosis de MDMA neurotóxicas para las neuronas serotoninérgicas (Seiden y Ricaurte, 1987; Gibb et al., 1994; Lew et al., 1997). Sin embargo, un estudio reciente en monos y babuinos indica que, bajo ciertas condiciones, la MDMA puede ser también una potente neurotoxina dopaminérgica(Ricaurte et al., 2002). En especial, cuando se daba MDMA a primates no humanos utilizando dosis de droga según los patrones del consumo recreativo habitual en humanos $(2 \mathrm{mg} / \mathrm{kg}$ cada 3 horas $\times 3$, en una dosis total de $6 \mathrm{mg} / \mathrm{kg}$ ), las neuronas cerebrales DA se veían seriamente afectadas (Ricaurte et al., 2002), además de las neuronas 5-HT. Esta observación indica que al recortar el intervalo de dosificación, el perfil neurotóxico de MDMA varía de una neurotoxina 5-HT selectiva a una neurotoxina conjunta 5-HT/DA.

A pesar de que los resultados en animales tratados con MDMA han demostrado que el consumo en humanos podría producir daños neurotóxicos, este hecho no ha disuadido, obviamente, a muchos individuos de consumir éxtasis con fines recreativos. Al contrario que en los animales, en los que es posible una evaluación directa del estado de las neuronas, existen pocos métodos para evaluar el estado de las poblaciones específicas de neuronas en un cerebro humano vivo. Sin embargo, los avances recientes en las técnicas de neuroimagen son prometedoras en este campo, siendo los únicos metódos de medición de la estructura y función cerebral en humanos vivos. El objetivo de este capítulo es analizar la idoneidad y validez de algunos de los más recientes métodos de neuroi- magen para detectar a largo plazo la neurotoxicidad 5-HT y DA inducida por la MDMA en cerebros vivos. Se discutirán los datos preclíncos y los clínicos disponibles hasta la fecha.

\section{TÉCNICAS DE NEUROIMAGEN}

\section{Tomografía Computarizada (TAC) e Ima- gen por Resonancia Magnética (MRI)}

La llegada de las técnicas de tomografía computarizada (TAC) e imagen por resonancia magnética (MRI) revolucionaron el campo de la neurorradiología al permitir la evaluación directa de las estructuras cerebrales en humanos vivos. Cada una de estas técnicas constituye una herramienta fiable y sensible en la detección de lesiones estructurales en el cerebro, tales como las asociadas a accidentes cerebrovasculares, tumores o infecciones. Siendo unas herramientas clínicas y de diagnóstico muy valoradas en grandes anomalías estructurales, no tienen, sin embargo, la sensibilidad necesaria para detectar pérdidas en poblaciones específicas neuronales, (por ejemplo, la neurotoxicidad 5HT o DA inducida por MDMA), ya que estas poblaciones específicas representan una fracción muy pequeña del parenquima cerebral (menos de un 1\%). Por ello, ni la TAC ni la MRI pueden utilizarse para valorar la neurotoxicidad selectiva inducida por MDMA en animales o humanos

\section{Tomografía por emisión de Positrones (PET)}

Estudios de densidad del transportador de serotonina (SERT): la capacidad de la PET utilizando un ligando neuronal específico para detectar la neurotoxicidad inducida por drogas en una población neuronal específica se indicó por primera vez en 1985, cuando Calne et al (1985) mostraron déficit dopaminérgico en adictos a la heroína que, sin querer, habían consumido heroína contaminada con MPTP, una potente neurotoxina dopaminérgica. Sin embargo, a pesar de los esfuerzos de una 
serie de grupos de investigadores, hasta esta última década no se consiguieron métodos para evaluar directamente el estado de las neuronas 5-HT en cerebros humanos vivos. En 1992, se informó sobre el desarrollo del $\left[{ }^{11} \mathrm{C}\right] \mathrm{McN} 5652$, un radioligando que marcaba selectivamente el SERT en vivo (Suehiro el at., 1993) A lo largo de los siguientes años, se estableció la validez del [" $\mathrm{C}] \mathrm{McN} 5652$ para utilización en humanos. En particular, estudios de validación en babuinos demostraron que utilizando la PET con un radioligando que se une al transportador de $5-\mathrm{HT},\left[{ }^{11} \mathrm{C}\right]$ McN5652 (Mc Cann et al, 1998), era posible detectar descensos en los transportadores de 5-HT marcados con $\left[{ }^{11} \mathrm{C}\right] \mathrm{McN} 5652$ en babuinos vivos, indicando, consecuentemente, una neurotoxicidad serotoninérgica inducida por MDMA (Scheffel et al., 1998).

A los estudios de validación en babuinos les siguieron pronto otros de PET con $\left[{ }^{11} \mathrm{C}\right]$ McN5652 en una cohorte de individuos que habían utilizado éxtasis en al menos 25 diferentes ocasiones previas, pero que habían mostrado su acuerdo en abstenerse del consumo de drogas psicoactivas a lo largo de, al menos, las dos semanas previas al estudio. Como en el caso de los babuinos tratados con MDMA, en los humanos expuestos a MDMA se detectaron descensos en la unión del $\left[{ }^{11} \mathrm{C}\right]$ McN5652. (McCann et al., 1998). Además, las reducciones en la unión específica del $\left[{ }^{11} \mathrm{C}\right] \mathrm{McN} 5652$ se correlacionaban con el alcance de la exposición previa al MDMA. A pesar del éxito inicial en el empleo de la PET para detectar la neurotoxicidad serotoninérgica inducida por la MDMA, los resultados quedaron en cierta manera comprometidos por los métodos utilizados en los primeros estudios de $\left[{ }^{11} \mathrm{C}\right] \mathrm{McN} 5652$. En especial, el método HPLC empleado en el momento de realización de estos estudios conducía a una elevada variabilidad en el cálculo del volumen de distribución aparente (DV). Por otra parte, este incremento de la variabilidad puede asociarse con una reducción de la capacidad para detectar diferencias entre grupos y, potencialmente, generar una subestimación de las diferencias entre consumidores de éxtasis y grupos de control. Desde ese momento se han realizado modificaciones en los métodos HPLC (Hilton et al., 2000) los cuales, en combinación con las mejoras en la tecnología de cámara PET, deberían permitir la confirmación y extensión de los primeros resultados. Especialmente, un estudio reciente de Burchert et al (2003) ha reproducido el hallazgo de una pérdida significativa de la unión específica $\left[{ }^{11} \mathrm{C}\right] \mathrm{McN} 5652$ en consumidores activos de MDMA, con una aparente recuperación en los siguientes meses. No obstante, debe señalarse que este estudio puede estar comprometido por el método utilizado para determinar la unión específica, dado que se utilizó el cerebelo como región "de referencia", (el cual, en una situación óptima, debería estar desprovisto de sitios SERT para ser idóneo como referencia). Como el cerebelo contiene sitios de unión SERT que, se sabe, pueden verse afectados por el éxtasis, la pérdida de sitios SERT del cerebelo, si es proporcional a la que ocurre en otros lugares cerebrales, puede llevar a resultados incorrectos o falsos negativos.

Recientemente se ha desarrollado un radioligando SERT alternativo, [" $\mathrm{C}] 3-$ amino-4(2-dimetilamino-metilfenilsulfanil)benzonitrilo o [ [ ${ }^{11}$ C]DASB, que también parece puede llegar a ser utilizado como radiotrazador SERT en PET (Wilson et al., 2000; Houle et al., 2000). Estudios preclínicos indican que, como el $\left[{ }^{11} \mathrm{C}\right](+)$ McN5652, el $\left[{ }^{11} \mathrm{C}\right] \mathrm{DASB}$ cuenta con una afinidad y selectividad para el SERT in vitro y que, in vivo, muestra unión saturable y específica al SERT en roedores. También, el daño en terminales axónicos serotoninérgicos por la neurotoxina p-cloroanfetamina ( $P C A)$, se asocia a pérdidas significativas en la unión [ $\left.{ }^{11} \mathrm{C}\right] \mathrm{DASB}$, in vivo, en roedores (Wilson et al., 2000). Estudios preclínicos de validación en babuinos comparando [ $\left[{ }^{11} \mathrm{C}\right] \mathrm{McN} 5652$ y $\left[{ }^{11} \mathrm{C}\right] \mathrm{DASB}$ en la detección de la neurotoxicidad 5-HT inducida por MDMA concluyen que ambos radioligandos pueden detectar pérdida de SERT inducida por MDMA y que cada ligando tiene ciertas ventajas (Szabo et al., 2002). En especial, mientras que el [" ${ }^{11} \mathrm{C}$ DASB tiene un mayor 
ratio señal-ruido en las regiones subcorticales con una mayor densidad de SERT, el $\left[{ }^{11} \mathrm{C}\right]$ McN5652 parece haber incrementado la capacidad para detectar la pérdida de SERT inducida por MDMA en regiones corticales y del cerebelo con menor densidad SERT. Estos resultados sugieren que el [ $\left.{ }^{11} \mathrm{C}\right] \mathrm{DASB}$ tiene un importante potencial para evaluar el estado del SERT cerebral en humanos (Szabo et al., 2002). Notablemente, estudios PET en humanos (Houle et al., 2000) muestran que el $\left[{ }^{11} \mathrm{C}\right] \mathrm{DASB}$ tiene unas características más apropiadas para evaluar el estado del SERT en escenarios clínicos y que el [ $\left.{ }^{11} \mathrm{C}\right] \mathrm{DASB}$ puede detectar con éxito la ocupación SERT con paroxetina y citalopram (Meyer et al., 2001). En la actualidad, están siendo desarrollados estudios con el $\left[{ }^{11} \mathrm{C}\right] \mathrm{DASB}$ en una cohorte de consumidores de MDMA.

Estudios de densidad del transportador de dopamina (DAT): Hasta el momento no se han publicado estudios PET o SPECT dirigidos a detectar neurotoxicidad DA en animales o humanos tratados con dosis de MDMA neurotóxicas para neuronas dopaminérgicas. Es de interés el informe remitido por Reneman et al (2002) donde se indica que consumidores de MDMA que también utilizan anfetaminas tienen una densidad reducida del DAT en el cuerpo estriado, mediante mediciones con $\left[{ }^{123} \mid\right]$ beta-CIT e imagen SPECT. Dado que el $\left[{ }^{123}\right]$ beta-CIT marca tanto los DAT como los SERT, y dado que se conoce que la anfetamina es tóxica para las neuronas DA, no queda claro si los efectos observados se deben al consumo de MDMA. Sin embargo, existen evidencias de que, dada la eficacia de una variedad de ligandos PET para axones DA (por ejemplo, el $\left[{ }^{11} \mathrm{C}\right]$ WIN 35,428, que se une al DAT ó el [ $\left.{ }^{11} \mathrm{C}\right] \mathrm{DTBZ}$, que marca transportadores-tipo 2 de monoamina vesicular VMAT2-), los estudios que validen el uso del PET para detectar la neurotoxicidad DA inducida por MDMA verán pronto la luz.

Metabolismo cerebral regional de glucosal: Estudios recientes con PET, utilizando 2-[8 $\left.{ }^{18}\right]-$ fluoro-2-deoxi-d-glucosa (FDG), han investigado la posibilidad de que el consumo de MDMA provoque alteraciones en el metabolismo cerebral regional de glucosa (Obrocki et al., 1999; Buchert et al., 2001; Obrocki et al., 2002). El estudio piloto original (Obrocki et al., 1999) sugería la alteración del metabolismo de la glucosa en consumidores de éxtasis. Dicho estudio se llevó a cabo en 93 consumidores de éxtasis y 27 individuos con un historial sin abuso conocido de drogas ilegales. Los resultados mostraron una reducción de la captación de FDG en el striatum y amígdala de los consumidores de éxtasis, en comparación con el grupo control. Se constató una correlación positiva entre la captación de FDG en la corteza cingulada y el tiempo que había pasado desde la última ingesta de éxtasis, y se observó que la captación cingulada de FDG variaba de acuerdo a la proximidad al consumo del éxtasis. Los cambios en la captación de FDG eran mayores en los consumidores de MDMA que habían comenzado a utilizarla a una edad más temprana.

La naturaleza de las alteraciones de los patrones del metabolismo cerebral regional de glucosa en consumidores de MDMA no está del todo clara, y puede o no ser indicativa de cambios neurotóxicos. A este respecto sería útil realizar estudios con FDG en primates no humanos, tratados con dosis neurotóxicas de MDMA, en los que se pudiera determinar la relación entre las alteraciones en marcadores axonales de neuronas 5-HT (y/o DA) y los cambios producidos en la captación de FDG. Resulta interesante que Sherkey et al (2000), utilizando una técnica autorradiográfica de tipo cuantitativo con $\left[{ }^{14} \mathrm{C}\right]$ deoxiglucosa, en ratas, hayan demostrado que las alteraciones permanentes en la utilización de glucosa hipocámpica tras una exposición repetida a MDMA, están asociadas con déficits serotoninérgicos.

Estudios de flujo sanguíneo con $\mathrm{H}_{2}{ }^{15} \mathrm{O}$ : Chang et al, evaluaron a 21 consumidores recreativos de éxtasis abstinentes y a un número similar de individuos de la misma edad y sexo (grupo control), utilizando técnicas SPECT y MRI, sin que se encontrasen diferencias significativas en el flujo sanguíneo 
cerebral global (CBF) o regional (rCBF). Otro estudio ha medido el rCBF, en consumidores de MDMA, durante la activación cognitiva ante una tarea de atención utilizando la PET y $\left[\mathrm{H}_{2}{ }^{15} \mathrm{O}\right.$ ] (Gamma et al., 2001). El fundamento de este estudio se basaba en que el déficit cognitivo que aparecía en consumidores abstinentes de MDMA podía estar asociado con patrones anormales de flujo sanguíneo cerebral. En este estudio no se encontraron diferencias entre los patrones de activación de los consumidores de MDMA y de los controles. Sin embargo, conviene resaltar que, tal como ocurre en los estudios de FDG descritos anteriormente, no se conoce si se producen alteraciones en los patrones de flujo sanguíneo por daños neurotóxicos, ya que no se han llevado a cabo estudios de validación. Es también importante tener en cuenta que la PET y $\left[\mathrm{H}_{2}{ }^{15} \mathrm{O}\right]$ puede no ser idónea para la detección de los rápidos cambios neuronales que ocurren durante el desarrollo de las tareas cognitivas. Además, la tarea utilizada en este estudio (por ejemplo, una tarea de atención) no está entre las que se encuentran alteradas en los consumidores de éxtasis.

\section{Tomografía Computarizada por Emisión de Fotón Simple (SPECT)}

Estudios de neurotranportadores: Se han realizado también estudios SPECT para intentar marcar el SERT en consumidores de MDMA (Semple et al., 1999; Reneman et al., $2001 \mathrm{a}, 2001 \mathrm{~b})$. El primer estudio, Ilevado a cabo por Semple et al, informó sobre disminuciones en la unión SERT de la región cortical, utilizando SPECT con [123] ] ヨ-CIT. Un estudio posterior de Reneman et al (2001a) que utilizaba el mismo radioligando, puso de manifiesto, en relación con un grupo control, una densidad media cortical más baja del SERT marcado con [123I] - CIT, en consumidores recientes de MDMA, pero no en aquellos que se habían mantenido abstinentes por más de un año. El mismo equipo también informó en otro estudio (Reneman et al., 2001 b), que el sexo y la duración de la exposición previa a la MDMA también parecían influir en los ratios de unión [ ${ }^{123}$ I] $\exists$ - CIT. Su conclusión fue que una mayor exposición a MDMA estaba asociada a disminuciones en la unión $\left[{ }^{123}\right.$ ] ] $\exists$ - CIT y que ésto ocurría más en mujeres que en varones.

A pesar de que los resultados del SPECT parecen apoyar la idea de que el éxtasis induce neurotoxicidad 5-HT en los consumidores humanos, estos resultados deben ser contemplados con cierta prudencia dado que, aunque esta técnica es capaz de medir la densidad SERT en el cerebro medio (Reneman et al., 2002), no está aun claro si el $\left[{ }^{123} \mid\right]$ ]- CIT puede medir correctamente uniones específicas a sitios SERT de la corteza, ya que estudios en primates no humanos han demostrado una ausencia de cambios en el nivel de la unión [ $\left.{ }^{123} \mid\right]$ ] $\exists$ - CIT en la corteza tras la administración del inhibidor de recaptación de serotonina, citalopram. Al igual que ocurría en el caso de los estudios con PET que utilizan 2-[ $\left.{ }^{18} \mathrm{~F}\right]$-fluoro-2-deoxi-d-glucosa y $\left[\mathrm{H}_{2}{ }^{15} \mathrm{O}\right]$, para dar respuesta a sí los daños 5-HT en la corteza cerebral inducidos por MDMA pueden ser detectados por SPECT con [123|] $]$ - CIT debería validarse este método. Se considera especialmente necesario desarrollar estudios, a ser posible con un antes y un después de utilización del éxtasis, con grandes primates no humanos, tales como los babuinos, que presentan unas distribuciones y densidades SERT corticales similares a los humanos.

Receptores 5- $\mathrm{HT}_{2 \mathrm{~A}}$ postsinápticos: Reneman et al (2000) midieron la densidad de los receptores $5-\mathrm{HT}_{2 \mathrm{~A}}$ en la corteza cerebral de consumidores de MDMA en un estudio preliminar cuyo objetivo era investigar la posibilidad de que el consumo de MDMA llevará a alteraciones compensatorias en los receptores $5-\mathrm{HT}_{2 \mathrm{~A}}$ postsinápticos y determinar si existía una relación entre la densidad de receptores 5- $\mathrm{HT}_{2 \mathrm{~A}}$ y los trastornos de memoria detectados en los consumidores de MDMA. Datos del estudio mostraron la existencia de ratios de unión [ ${ }^{123}$ ] -5-I-R91150 ligeramente superiores en la corteza occipital en usuarios de MDMA frente a los del grupo control y esto es interpretado por los investigadores como una indicación de regulación a 
la alza. Se encontró que la media de unión a receptores $5-\mathrm{HT}_{2 A}$ corticales se correlacionaba positivamente con las medidas de memoria en los consumidores de MDMA, sugiriendo una relación potencial entre la función de la memoria y las alteraciones en la función del receptor $5 \mathrm{HT}_{2 \mathrm{~A}}$.

Aunque sugestivos, estos resultados, tal como indican los investigadores, deben ser considerados preliminares, dado el bajo número de individuos incluidos en el estudio. Los estudios de validación podrán ser útiles en la clarificación de la naturaleza de los cambios en el receptor $5-\mathrm{HT}_{2 A}$ en los consumidores de MDMA, ya que los estudios previos no indican que existan cambios permanentes en los receptores $5-\mathrm{HT}_{2 A}$ en el marco de la neurotoxicidad 5-HT inducida por MDMA. (Scheffel et al., 1992).

\section{Imagen por Resonancia Magnética Funcio- nal (fMRI)}

Hasta el momento no se han publicado estudios que impliquen la utilización de fMRI en consumidores de MDMA. Este método de neuroimagen permite la detección de los cambios en tiempo real en el flujo sanguíneo cerebral y ha llevado a importantes avances en la comprensión de las regiones cerebrales que tienen que ver con una gran variedad de procesos cognitivos cerebrales (Brett et al., 2002). Dado que se ha demostrado que los consumidores de MDMA presentan un déficit en la función cognitiva (Parrott, 1998; 2002), este método puede abrir las puertas para aclarar los sustratos neurobiológicos de este déficit.

\section{Imagen por Resonancia Magnética de Di- fusión y Perfusión}

La resonancia magnética de difusión es un método de neuroimagen que permite obtener medidas cuantitativas del movimiento difusional de las moléculas de agua en un tejido biológico. Procesos que llevan a una alteración de los elementos estructurales del tejido llevan a un incremento del coeficiente de difusión aparente (ADC). Por lo tanto, este método puede llegar a ser utilizado en el estudio de cambios del tejido en diversas formas de daño cerebral o degeneración. Una técnica relacionada, la resonancia magnética de perfusión de contraste-realce dinámico permite la evaluación de la vascularización cerebral mediante el cálculo de mapas de volumen sanguíneo cerebral relativo (rCBV), que pueden estar también alterados en distintos tipos de daño cerebral. Únicamente existe un estudio piloto que utilice la resonancia magnética de difusión y perfusión para detectar posibles anomalías en los consumidores de MDMA (Reneman et al., 2001). Los investigadores hallaron que los valores del coeficiente de difusión aparente (ADC) eran significativamente más elevados en el globus pallidus de los consumidores de MDMA que en los de los no consumidores. Incrementos en el volumen cerebral relativo ( $\mathrm{rCBV}$ ) del pallidus se correlacionaban positivamente con la extensión de la exposición al consumo previo de MDMA. El incremento en los valores ADC se interpretó como una posible evidencia de daño axónico, mientras que el incremento del rCBV en el pallidus de consumidores de MDMA se cree que está asociado a un incremento del flujo sanguíneo. A pesar de que puede resultar interesante, la naturaleza de estos hallazgos no está clara ya que todavía no se conoce si la resonancia magnética de difusión y/o perfusión tiene la suficiente sensibilidad para detectar los daños neurotóxicos 5-HT o DA inducidos por la MDMA. Es más, aun no está claro si la señal producida por daños en estas poblaciones neuronales específicas es lo suficientemente amplia como para ser detectada con este método. A este respecto sería de gran utilidad realizar estudios con primates no humanos con una distribución de fibras nerviosas monoaminérgicas similares a las de los humanos (tratamiento pre y post con MDMA).

\section{Imagen por Tensor de Difusión (DTI)}

La Imagen por Tensor de Difusión (DTI) es una forma avanzada de Resonancia Magnéti- 
ca de Difusión que permite la visualización de la anisotropía (dirección) del movimiento del agua causado por la presencia de axones, cubiertas axonales, celulas glia, y vasculatura (Yamada et al., 2003). Las imágenes obtenidas con este método pueden ser reconstruidas para rastrear tridimensionalmente la orientación de las fibras macroescópicas en el cerebro. Como la neurotoxicidad MDMA se caracteriza por una degeneración axonal, este método puede ser útil en la detección del daño a las neuronas 5-HT o DA inducida por MDMA. Hasta el momento no existen estudios preclínicos o clínicos que hayan utilizado DTI para detectar la neurotoxicidad inducida por MDMA.

\section{Resonancia Magnética Espectroscópica}

La resonancia magnética espectroscópica de protón ( ${ }^{H} \mathrm{H}$ MRS) ha sido empleada por muchos equipos de investigadores para detectar anomalías neuroquímicas en consumidores de MDMA (Chang et al., 1999; Reneman et al., 2001, 2002; Obergreisser et al., 2001). El método se basa en una técnica sensible y no invasiva que permite medir las concentraciones cerebrales de $\mathrm{N}$-acetilaspartate [NA], un marcador neuronal, y mio-inositol [MI], un tentativo marcador glial. El fundamento para utilizar ${ }^{1} \mathrm{H}$ MRS en consumidores de MDMA se basa en que disminuciones en NA o incrementos en $\mathrm{MI}$ podrían representar la pérdida de vitalidad neuronal o activación glial, respectivamente.

Tres de los cuatro estudios llevados a cabo hasta el momento (Chang et al., 1999; Reneman et al., 2001, 2002) han encontrado alteraciones en las medidas de ${ }^{1} \mathrm{H}$ MRS en los consumidores de MDMA en comparación con los grupos control. En el estudio de Chang et al, la exposición a MDMA se asoció con reducciones en el ratio $\mathrm{Ml} /$ creatinina de la materia blanca parietal, existiendo una correlación positiva, entre el ratio $\mathrm{Ml}$ /creatinina y la extensión del consumo anterior de MDMA, en la materia blanca parietal y en la corteza occipital. Por el contrario, los dos estudios de Reneman et al encontraron dis- minuciones en los ratios de NA/creatinina o $\mathrm{NA}$ /colina en la corteza pre-frontal y/o frontal. Existía una fuerte correlación inversa entre los ratios en la corteza frontal y la función cognitiva y ello sugería que la neuroquímica alterada podía estar relacionada con una función cognitiva alterada en los consumidores de MDMA.

Los métodos ${ }^{1} \mathrm{H}$ MRS resultan claramente prometedores a la hora de poder detectar anomalías neuronales en seres humanos vivos. Como muchas de las nuevas técnicas de neuroimagen no tienen, sin embargo, una validación como medida para detectar el daño neurotóxico inducido por MDMA. Como los axones 5-HT y DA representan una fracción muy pequeña de los elementos neuronales en las regiones cerebrales donde se han observado cambios MRS, no está claro si los perfiles neuronales anormales encontrados en los consumidores de MDMA están relacionados con la neurotoxicidad o con otros procesos. Por ejemplo, como los cambios en NA se han detectado en diferentes situaciones, no se puede considerar que los cambios MRS sean un indicador específico de neurotoxicidad serotoninérgica o dopaminérgica. Como en el caso del resto de modalidades de neuroimagen, para definir la naturaleza neurobiológica de los cambios MRS en consumidores de MDMA sería de gran utilidad la realización de estudios preclínicos en primates no humanos con neurotoxicidad inducida por MDMA documentada (antes y después del tratamiento de MDMA).

\section{CONCLUSION}

Existe una clara evidencia de que el MDMA produce daños neurotóxicos serotoninérgicos en una gran variedad de especies animales, incluidos los primates no humanos. Datos recientes en monos y babuinos indican que cuando la MDMA es suministrado en intervalos cortos y regulares, utilizando una dosificación similar a la consumida por usuarios recreativos de éxtasis, es capaz también 
de producir daños dopaminérgicos. Algunas de las más recientes técnicas de neuroimagen resultan prometedoras para averiguar si los consumidores humanos de MDMA son, como los animales, sensibles a la neurotoxicidad 5-HT y DA inducida por MDMA. Actualmente, sólo los métodos PET y SPECT que utilizan radioligandos específicos para el SERT, han sido validados como capaces de detectar daños 5-HT inducidos por MDMA, y no se han publicado estudios que prueben la potencial neurotoxicidad DA inducida por MDMA en humanos. Sin embargo, varios equipos de investigadores han empleado algunas de las más recientes técnicas de neuroimagen para probar posibles anomalías en consumidores de MDMA, incluyendo PETFDG y $\mathrm{H}_{2} \mathrm{O}^{15}$; SPECT utilizando [ ${ }^{123} \mid$ ] ß-CIT y [23I]-5-I-R91150, 'H MRS; y MRI de Difusión y Perfusión. La MRI Funcional también parece prometedora en el estudio de la neurotoxicidad inducida por MDMA, especialmente para aclarar los correlatos neurobiológicos de las anomalías cognitivas identificadas en consumidores de MDMA.

Aunque cada una de las nuevas técnicas de neuroimagen resulta prometedora para detectar la neurotoxicidad por MDMA en humanos, es también necesario llevar a cabo estudios preclínicos para validar cada uno de los métodos. Estos estudios preclínicos, que permiten una evaluación directa del tejido cerebral en paralelo a la evaluación de los métodos de neuroimagen, darán las bases para que los datos procedentes de las técnicas de neuroimagen sean interpretados correctamente. Por ejemplo, si se determina que ${ }^{1} \mathrm{H}$ MRS no tiene la capacidad requerida para detectar la neurotoxicidad 5-HT o DA inducida por MDMA, los cambios detectados en los consumidores de MDMA deberán ser interpretados como representativos de una disfunción neuronal de otro tipo. Un razonamiento similar podrá ser aplicado para cada una de las técnicas de neuroimagen en la que los consumidores de MDMA presentan diferencias en relación a los grupos de control, pero con las cuales, aún, no se ha podido demostrar un daño neurotóxico 5-HT o DA específico.

Las pasadas décadas se han caracterizado por importantes avances en nuestra capacidad para evaluar el estado del cerebro humano vivo. Cada uno de los métodos de neuroimagen descritos aquí resultan prometedores en el avance de nuestra comprensión de las funciones cerebrales humanas normales y sus patologías. La investigación en neuroimagen durante la próxima década permitirá alcanzar resultados que aclararán la naturaleza y extensión de la neurotoxicidad inducida por MDMA en humanos. Estos estudios, reforzados por estudios preclínicos utilizando métodos paralelos, permitirán también arrojar la luz sobre diferentes procesos cerebrales normales y con disfuncionalidades.

\section{Agradecimientos}

Agradecemos a C. Connelly por su ayuda en la preparación del documento. Este trabajo ha sido financiado por las becas USPHS DA 5707, DA 13790, DA 09487, DA 00206, and DA 10217.

\section{REFERENCIAS}

Brett M, Johnsrude IS, Owen AM. The problem of functional localization in the human brain. Nat Rev Neurosci 2002; 3: 243-249.

Buchert R, Thomasius R, Nebeling B, Petersen K, Obrocki J, Jenicke L, Wilke F, Wartberg L, Zapletalova P, Clausen M. Long-Term effects of "Ecstasy" use on serotonin transporters of the brain investigated by PET. J Nucl Med 2003; 44: 375-384.

Buchert R, Obrocki J, Thomasius R, Vaterlein O, Petersen K, Jenicke L, Bohuslavizki KH, Clausen M. Long-term effects of 'ecstasy' abuse on the human brain studied by FDG PET. Nucl Med Commun 2001; 22: 889-897.

Calne DB, Langston JW, Martin W, Stoessel A, Ruth T, Adam M, Schulzer M. Positron emission tomography after MPTP: observations relating to the cause of Parkinson's disease. Nature 1985; 317: 246-248. 
Chang L, Grob CS, Ernst T, Itti L, Mishkin FS, JoseMelchor R, Poland RE. Effect of ecstasy [3,4methylenedioxymethamphetamine (MDMA)] on cerebral blood flow: a co-registered SPECT and MRI study. Psychiatry Res 2000; 98: 1528.

Chang L, Ernst T, Grob CS, Poland RE. Cerebral (1)H MRS alterations in recreational 3,4-methylenedioxymethamphetamine (MDMA, "ecstasy") users. J Magn Reson Imaging 1999; 10: 521526.

Christophersen AS. Amphetamine designer drugs - an overview and epidemiology. Toxicol Lett 2000; 112-113: 127-131.

Gamma A, Buck A, Berthold T, Vollenweider FX. No difference in brain activation during cognitive performance between ecstasy (3,4-methylenedioxymethamphetamine) users and control subjects: a [H2(15)O]-positron emission tomography study. J Clin Psychopharmacol 2001; 21: 66-71.

Hilton J, Yokoi F, Dannals RF, Ravert HT, Szabo Z, Wong DF. Column-switching HPLC for the analysis of plasma in PET imaging studies. Nucl Med Biol 2000; 27: 627-630.

Houle S, Ginovart N, Hussey D, Meyer JH, Wilson AA. Imaging the serotonin transporter with positron emission tomography: initial human studies with [ $\left.{ }^{11} \mathrm{C}\right] \mathrm{DAPP}$ and $\left[{ }^{11} \mathrm{C}\right] \mathrm{DASB}$. Eur $\mathbf{J}$ Nucl Med 2000; 27: 1719-1722.

Johnston LD, O'Malley PM, Bachman JG. Monitoring the Future national survey results on drug use, 1975-2000. Volume I: Secondary school students (NIH Publication No. 014924). Bethesda, MD: National Institute on Drug Abuse, 2001a.

Johnston LD, O'Malley PM, Bachman JG. Monitoring the Future national survey results on drug use, 1975-2000. Volume 2: College students and adults ages 19-40 (NIH Publication No. 01-4925). Bethesda, MD: National Institute on Drug Abuse, 2001b.

Lew R, Malberg JE, Ricaurte GA, Seiden LS. Evidence for and mechanism of action of neurotoxicity of amphetamine related compounds. En: Highly Selective Neurotoxins: Basic and Clinical Applications, Kostrzewa RM, (ed), Humana Press Inc., Totowa, pp. 235-268, 1997.

McCann UD, Ricaurte GA, Molliver ME. "Ecstasy" and serotonin neurotoxicity: new findings raise more questions. Arch Gen Psychiatry 2001; 58: 907-908.
McCann UD, Szabo Z, Scheffel U, Dannals RF, Ricaurte GA. Positron emission tomographic evidence of toxic effect of MDMA ("Ecstasy") on brain serotonin neurons in human beings. Lancet 1998; 352: 1433-1437.

Meyer JH, Wilson AA, Ginovart N, Goulding V, Hussey D, Hood K, Houle S. Occupancy of serotonin transporters by paroxetine and citalopram during treatment of depression: a $\left[{ }^{11} \mathrm{C}\right] \mathrm{DASB}$ PET imaging study. Am J Psychiatry 2001; 158: 1843-1849.

Obergriesser T, Ende G, Braus DF, Henn FA. Hippocampal 1H-MRSI in ecstasy users. Eur Arch Psychiatry Clin Neurosci 2001; 251: 114-116.

Obrocki J, Schmoldt A, Buchert R, Andresen B, Petersen K, Thomasius R. Specific neurotoxicity of chronic use of ecstasy. Toxicol Lett 2002; 127: 285-297.

Obrocki J, Buchert R, Vaterlein O, Thomasius R, Beyer W, Schiemann T. Ecstasy--long-term effects on the human central nervous system revealed by positron emission tomography. $\mathbf{B r}$ J Psychiatry 1999; 175:186-188.

Parrott AC, Lees A, Granham NJ, Jones M, Wesnes $K$. Cognitive performance in recreational users of MDMA or "ecstasy": evidence for memory deficits. J Psychopharmacol 1998; 12: 79-83.

Parrott AC, Buchanan T, Scholey AB, Heffernan T, Ling J, Rodgers J. Ecstasy/MDMA attributed problems reported by novice, moderate and heavy recreational users. Hum Psychopharmacol 2002; 17: 309-312.

Reneman L, Booij J, Majoie CB, Van Den Brink W, Den Heeten GJ. Investigating the potential neurotoxicity of Ecstasy (MDMA): an imaging approach. Hum Psychopharmacol 2001; 16 : 579-588.

Reneman L, Booij J, Habraken JB, De Bruin K, Hatzidimitriou G, Den Heeten GJ, Ricaurte GA. Validity of [123I]beta-CIT SPECT in detecting MDMA-induced serotonergic neurotoxicity. Synapse 2002; 46: 199-205.

Reneman L, Booij J, Lavalaye J, de Bruin K, Reitsma JB, Gunning B, den Heeten GJ, van Den Brink W. Use of amphetamine by recreational users of ecstasy (MDMA) is associated with reduced striatal dopamine transporter densities: a [123I]beta-CIT SPECT study--preliminary report. Psychopharmacology (Berl) 2002; 159: 335-340.

Reneman L, Endert E, de Bruin K, Lavalaye J, Feenstra MG, de Wolff FA, Booij J. The acute and chronic effects of MDMA ("ecstasy") on 
cortical 5-HT2A receptors in rat and human brain. Neuropsychopharmacology 2002; 26: 387-396.

Reneman L, Booij J, de Bruin K, Reitsma JB, de Wolff FA, Gunning WB, den Heeten GJ, van den Brink W. Effects of dose, sex, and longterm abstention from use on toxic effects of MDMA (ecstasy) on brain serotonin neurons. Lancet 2001; 358: 1864-1869.

Reneman L, Lavalaye J, Schmand B, de Wolff FA, van den Brink W, den Heeten GJ, Booij J. Cortical serotonin transporter density and verbal memory in individuals who stopped using 3,4methylenedioxymethamphetamine (MDMA or "ecstasy"): preliminary findings. Arch Gen Psychiatry 2001; 58: 901-906.

Reneman L, Majoie CB, Habraken JB, den Heeten GJ. Effects of ecstasy (MDMA) on the brain in abstinent users: initial observations with diffusion and perfusion MR imaging. Radiology 2001; 220: 611-617.

Reneman L, Booij J, Schmand B, van den Brink W, Gunning B. Memory disturbances in "Ecstasy" users are correlated with an altered brain serotonin neurotransmission. Psychopharmacology (Berl) 2000; 148: 322-324.

Reneman L, Habraken JB, Majoie CB, Booij J, den Heeten GJ. MDMA ("Ecstasy") and its association with cerebrovascular accidents: preliminary findings. AJNR Am J Neuroradiol 2000; 21: 1001-1007.

Ricaurte GA, Yuan J, McCann UD. (") 3,4-Methylenedioxymethamphetamine (MDMA, "Ecstasy")-induced serotonin-neurotoxicity: Studies in animals. Neuropsychobiol 2000; 42: 5-10.

Ricaurte GA, Yuan J, Hatzidimitriou G, Cord Branden J, McCann Una D: Severe dopaminergic neurotoxicity in primates given typical recreational doses of MDMA ("Ecstasy"). Science 2002 ; 297: 2260-2263.

Ricaurte GA, McCann UD. Assessing long-term effects of MDMA (Ecstasy). Lancet 2001; 358: 1831-1832.

Ricaurte GA, McCann UD, Szabo Z, Scheffel U. Toxicodynamics and long-term toxicity of the recreational drug, 3,4-methylenedioxymethamphetamine (MDMA, 'Ecstasy'). Toxicol Lett 2000; 112-113: 143-146.

Rogers RD, Robbins TW. Investigating the neurocognitive deficits associated with chronic drug misuse. Curr Opin Neurobiol 2001; 11: 250257.
Scheffel U, Lever JR, Stathis M, Ricaurte GA. Repeated administration of MDMA causes transient down-regulation of $5-\mathrm{HT}_{2}$ receptors. Neuropharmacology 1992; 31: 881-893.

Scheffel U, Szabo Z, Mathews WB, Finley PA, Dannals RF, Ravert HT, Szabo K, Yuan J, Ricaurte GA. In vivo detection of short- and long-term MDMA neurotoxicity--a positron emission tomography study in the living baboon brain. Synapse 1998; 29: 183-192.

Schreckenberger M, Gouzoulis-Mayfrank E, Sabri O, Arning C, Tuttass T, Schulz G, Kaiser HJ, Wagenknecht G, Sass H, Bull U. [Investigations on the effect of "ecstasy" on cerebral glucose metabolism: an 18-FDG PET study]. Nuklearmedizin 1998; 37: 262-267.

Seiden LS, Ricaurte GA: Neurotoxicity of methamphetamine and related drugs. En: Psychopharmacology - A Generation of Progress, Meltzer HY (ed). Raven Press, NY, pp. 359-366, 1987.

Sharkey J, McBean DE, Kelly PA. Alterations in hippocampal function following repeated exposure to the amphetamine derivative methylenedioxymethamphetamine ("Ecstasy"). Psychopharmacology (Berl) 1991; 105: 113-118.

Semple DM, Ebmeier KP, Glabus MF, O'Carroll RE, Johnstone EC. Reduced in vivo binding to the serotonin transporter in the cerebral cortex of MDMA ('ecstasy') users. Br J Psychiatry 1999; 175: 63-69.

Suehiro M, Scheffel U, Dannals RF, Ravert HT, Ricaurte GA, Wagner HN Jr. A PET radiotracer for studying serotonin uptake sites: carbon-11McN-5652Z. J Nucl Med 1993; 34: 120-127.

Szabo Z, McCann UD, Wilson AA, Scheffel U, Owonikoko T, Mathews WB, Ravert HT, Hilton J, Dannals RF, Ricaurte GA. Comparison of (+)(11)C-McN5652 and (11)C-DASB as serotonin transporter radioligands under various experimental conditions. J Nucl Med 2002; 43: 678692.

Wilson AA, Ginovart N, Schmidt M, et al. Novel radiotracers for imaging the serotonin transporter by positron emission tomography: synthesis, radiosynthesis, and in vitro and ex vivo evaluation of (11)C-labeled 2-(phenylthio)araalkyl amines. J Med Chem 2000; 43: 3103-3110.

Yamada K, Kizu O, Mori S, Ito H, Nakamura H, Yuen S, Kubota T, Tanaka O, Akada W, Sasajima H, Mineura K, Nishimura T. Brain Fiber Tracking with Clinically Feasible Diffusion-Tensor MR Imaging: Initial Experience. Radiology 2003; 227: 295-301. 